\title{
TRANSFORMATION OF SINGULAR LIGHT BEAM TRANSVERSE STRUCTURE IN RESONANT MEDIA
}

\author{
O. G. Romanov ${ }^{*}$ and A. L. Tolstik
}

UDC 535.34

The peculiarities of transformation of transverse structure for singular light beams propagating in a resonant medium have been analyzed theoretically. The influence of nonlinear absorption, self-focusing, and defocusing on the characteristics of optical vortices with different topological structure has been studied. The formation conditions and parameters for singular spatially localized structures have been characterized.

Key words: singular optics, optical vortex, resonant medium, self-focusing, defocusing.

Introduction. A screw dislocation of a light-beam wave front appears as a screw surface with a special point at which the field amplitude is equal to zero and the phase is undefined [1]. A phase shift in multiples of $2 \pi$ occurs if this singularity is avoided. Light beams with wave-front dislocations (optical vortices) that are observed in optical fields with a complex transverse structure [2] and in laser cavities [3] or are produced as a result of light diffraction on computer synthesized holograms [4] are constantly of interest to researchers because of numerous potential applications.

The propagation of singular light beams in nonlinear media is investigated in order to find the stability conditions of their transverse and topological structure [5,6], to study the waveguide properties of transverse localized optical vortices in media with different types of nonlinearity [7-9], to analyze their polarization structure [10], and to develop methods for transforming the topological structure through nonlinear interactions [11, 12]. Transformation of the transverse structure of Bessel light beams in various nonlinear materials is widely investigated together with singular beams [13-15].

Herein features of the transformation of the transverse structure of singular light beams with the combined influence of nonlinear absorption and self-focusing (defocusing) of the radiation in resonant media are analyzed and the conditions for forming localized transverse structures as dark soliton vortices are determined. The model of resonant media that is used is suitable for describing the interaction of radiation with dye solutions and with activated crystals, crystals with color centers, and vapors of complex organic compounds.

Theoretical Model of the Propagation of Singular Light Beams in Resonant Media. The propagation of singular light beams in resonant media will be investigated using a truncated wave equation for the complex amplitude of field $E$ of the form [16]:

$$
2 i k \frac{\partial E}{\partial z}+\Delta_{\perp} E=-\frac{4 \pi \omega^{2}}{c^{2}} \chi_{\mathrm{nl}} E,
$$

where $k=\omega n_{0} / c$ is the wave vector; $\omega$, the light wave frequency; $n_{0}$, the initial index of refraction (determined by the solvent or material of the solid-state matrix); $\Delta_{\perp}=\partial^{2} / \partial x^{2}+\partial^{2} / \partial y^{2}$, the Laplacian cross section; $\chi_{\mathrm{nl}}=n_{0} \Delta \hat{n} / 2 \pi$, the nonlinear susceptibility of the medium; and, $\Delta \hat{n}$ is the light induced change of complex index of refraction of the resonant medium.

Considering the frequency of the active laser radiation $\omega$ to be close to the center of any absorption band of the compound $\omega_{12}$, we examine a two-level model of the medium. We start with the Kramers-Kronig relationship in order to describe the spectral dependence of the index of refraction that is due to a resonance mechanism of non-

\footnotetext{
${ }^{*}$ To whom correspondence should be addressed.
}

Belarusian State University, 4 Nezavisimosti Ave., Minsk, 220030, Belarus; e-mail: romanov@bsu.by. Translated from Zhurnal Prikladnoi Spektroskopii, Vol. 75, No. 4, pp. 509-515, July-August, 2008. Original article submitted April 30, 2008. 
linearity. This relates the real and imaginary parts of the complex index of refraction $\hat{n}=n+i \kappa[17]: n(\omega)=$ $\frac{1}{\pi} \int_{-\infty}^{\infty} \frac{\kappa\left(\omega^{\prime}\right)}{\left.\omega^{\prime}-\omega\right)} d \omega^{\prime}$, where $\kappa\left(\omega^{\prime}\right)$ is the spectral dependence of the extinction coefficient that can be represented in the examined model of a nonlinear medium as [17]: $\kappa(\omega)=\frac{\hbar c N}{2 v}\left[B_{12}(\omega)-n_{2}\left(B_{12}(\omega)+B_{21}(\omega)\right)\right], N$ is the number of absorbing centers per unit volume; $v=c / n_{0}$, the speed of light in the medium; $n_{2}=N_{2} / N$, the normalized value of the population of the second (excited) energy level. The spectral dependence of the index of refraction is determined by the expression $n(\omega)=\frac{\hbar c N}{2 v}\left[\Theta_{12}(\omega)-n_{2}\left(\Theta_{12}(\omega)+\Theta_{21}(\omega)\right)\right]$, where coefficients $\Theta_{i j}(\omega)$ are related by Kramers-Kronig relationships to Einstein coefficients $B_{i j}(\omega)$.

The nonlinear susceptibility of the resonant medium is expressed in terms of the change of complex index of refraction $\Delta \hat{n} \equiv \Delta n+i \Delta \kappa=-\frac{\kappa_{0} n_{2}}{B_{12}}\left(\hat{\Theta}_{12}+\hat{\Theta}_{21}\right)$. Here $\hat{\Theta}_{i j}=\Theta_{i j}+i B_{i j}$ and $\kappa_{0}=\frac{\hbar c N B_{12}}{2 v}$ is the linear extinction coefficient. Assuming the absorption and transmission curves coincide, $\Theta_{12}=\Theta_{21}$. Then the change of index of refraction of the resonant medium for steady-state excitation has the form: $\Delta n=-\kappa_{0} \frac{\Theta_{12}}{B_{12}} \frac{\alpha I}{1+\alpha I}$, where parameter $\alpha=\left(B_{12}+\right.$ $\left.B_{21}\right) / v P_{21}$ determines the saturation intensity of the resonant transition $\left(P_{21}\right.$ is the total probability of spontaneous and radiationless transitions).

We note that the nonlinear change of index of refraction for exact resonance $\left(\omega=\omega_{12}\right)$ reverts to zero $(\Delta n=0)$ and the action of the light beam on the resonant medium consists of a light-induced change of the absorption coefficient. If the laser radiation frequency is tuned to the short-wavelength part of the spectrum relative to the absorption band center $\left(\omega>\omega_{12}\right)$, the index of refraction of the medium is increased depending on the intensity of the light beam $(\Delta n>0)$. This determines if the diffraction dispersion and subsequent self-focusing can be compensated. However, if the radiation frequency is tuned to the long-wavelength part of the spectrum $\left(\omega<\omega_{12}\right)$, the change of index of refraction is negative $(\Delta n<0)$. This corresponds to defocusing of the light beam and its propagation in a nonlinear medium.

Considering the explicit form of the nonlinear susceptibility of a resonant medium $[18] \chi_{\mathrm{nl}}(\omega)=\frac{n_{0} \kappa_{0}}{2 \pi}$ $\times\left[\frac{\hat{\Theta}_{12}}{B_{12}}-\frac{\hat{\alpha} I}{1+\alpha I}\right]$, where the complex parameter of nonlinearity is determined by the expression $\hat{\alpha}=a+i \alpha=$ $\left(\hat{\Theta}_{12}+\hat{\Theta}_{21}\right) / v P_{12}$, the truncated wave equation (1) becomes:

$$
\frac{\partial E}{\partial z}+\frac{1}{2 i k} \Delta_{\perp} E=i \frac{k_{0}}{2}\left(\frac{\hat{\Theta}_{12}}{B_{12}} E+\frac{\hat{\alpha} I}{1+\alpha I} E\right)
$$

where $k_{0}=2 \omega \kappa_{0} / c$ is the linear absorption coefficient. The first term in the right part of the equation accounts for linear absorption and the phase advance; the second determines the effects of absorption saturation and the light-induced change of complex index of refraction of the resonant medium.

During numerical modeling of Eq. (2) it was assumed that a light beam directed at the boundary $z=0$ of the nonlinear medium has a time-invariant profile along the transverse coordinate and contains a vortex phase dislocation of topological charge $m: E(z=0, r, \varphi)=E_{0}\left[r / r_{0}\right]^{|m|} \exp \left[-\left(r / \sqrt{2} r_{0}\right)^{2}+i m \varphi\right]$. Figure 1 shows the structure of such light beams, where Fig. 1a-c show the transverse intensity distribution (maximum values are shown as white light); Fig. $1 \mathrm{a}^{\prime}-\mathrm{c}^{\prime}$, the phase distribution that changes from 0 (black light) to $2 \pi$ (white light). We note that the instance $m=0$ corresponds to a beam with a Gaussian intensity distribution and an initially planar wave front. The half-width of the light beam at the entrance of the nonlinear medium is set to $r_{0}=200 \mu \mathrm{m}$; peak intensity of the beam varied in the range $\alpha I_{0}=0.1-10$; radiation wavelength $\lambda=0.5 \mu \mathrm{m}$; radiation frequency tuning from the center of the absorption band $\delta=\left(\omega-\omega_{12}\right) / \Delta=0, \pm 2$, where $\Delta$ is the half-width of the Gaussian absorption curve and the initial absorption 
$a$
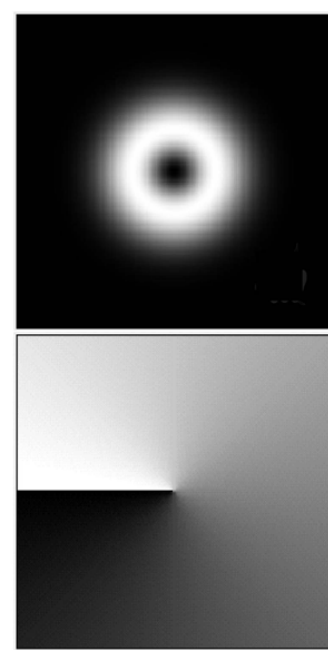

$a^{\prime}$ $b$
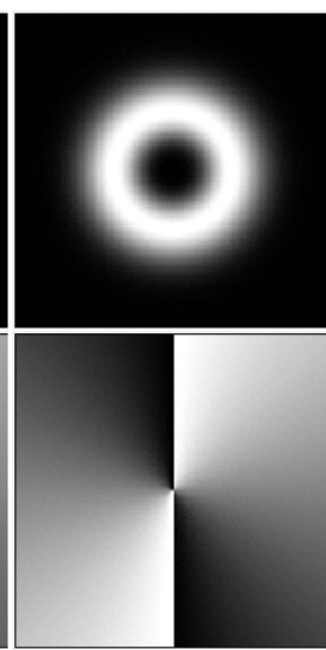

$b^{\prime}$
C

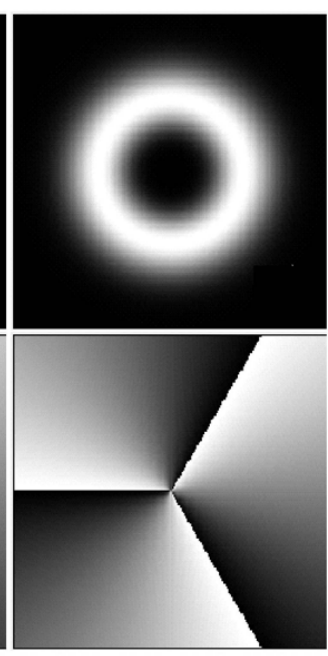

$C^{\prime}$

Fig. 1. Intensity distribution $(a-c)$ and wave front of optical vortices $\left(a^{\prime}-c^{\prime}\right)$ with topological charge $m=1\left(\mathrm{a}, \mathrm{a}^{\prime}\right), 2\left(\mathrm{~b}, \mathrm{~b}^{\prime}\right)$, and 3 (c, $\left.\mathrm{c}^{\prime}\right)$.

coefficient was taken as $k_{0}=0.05 \mathrm{~cm}^{-1}$. An absolutely stable two-step (three-layer) explicit method [19] that could follow the evolution of the light beam at distances of the order of the diffraction line $L_{\mathrm{d}}=2 \pi r_{0}^{2} / \lambda$ was used in the numerical calculations.

Discussion. Nonlinear absorption of singular light beams. It is well known [18] that the absorption coefficient of a resonant medium decreases with increasing intensity of the light beam because of a bleaching effect $\left[k(I)<k_{0}\right]$. This causes a deviation from the linear Buger absorption law. The linear (as a result of diffraction dispersion) and nonlinear (because of self-focusing or defocusing) change of beam transverse dimensions must also be considered in examining the interaction of light beams of finite transverse dimensions and complex structure with a resonant medium. For example, self-focusing increases the concentration of energy in the axial region for a Gaussian peak. This enhances saturation of the resonance transition and decreases the local absorption coefficient [20]. On the other hand, defocusing of the beam leads to its transverse spreading and an increased fraction of absorbed energy per unit area of beam.

The numerical experiments showed that these effects are more noticeable for singular light beams. Figure 2 shows the calculated change of light-beam power $P(z)=\frac{c n_{0}}{2 \pi} \int_{0}^{2 \pi} d \varphi \int_{0}^{\infty}|E(z, r, \varphi)|^{2} r d r$ containing a phase dislocation of the $m$-th order as a function of penetration depth into the resonant medium. We note that these functions were calculated for a single beam input power with different values of the topological order $\mathrm{m}$ and were normalized over $P(\mathrm{z})=$ $0)$. The transverse coordinate $z$ was normalized over the diffraction length of the light beam $L_{\mathrm{d}}$. It can be seen that the difference in the effectiveness of absorption of the Gaussian beam (curve 1) and singular beams (curves 2-4) is insignificant if the frequency of the laser radiation coincides with the center of the absorption band of the resonant medium (Fig. 2a) with the intensity of the light beams of the order of the saturation intensity of the resonant transition. However, the bleaching effect in the field of the Gaussian beam becomes more noticeable compared with the singular beams as the intensity increases (curves $1^{\prime}-4^{\prime}$ ).

Figure $2 \mathrm{~b}$ shows the calculations of the power change of singular beams for propagation in a medium with a defocusing resonant nonlinearity that occurs for a negative frequency detuning from the center of the resonant transition. The spatially heterogeneous saturation of the resonant transition is significantly smoother at the selected parameters of the nonlinear medium and radiation than in the first instance. This increases the effective absorption coefficient and draws the absorption curves closer to the linear Buger law. 

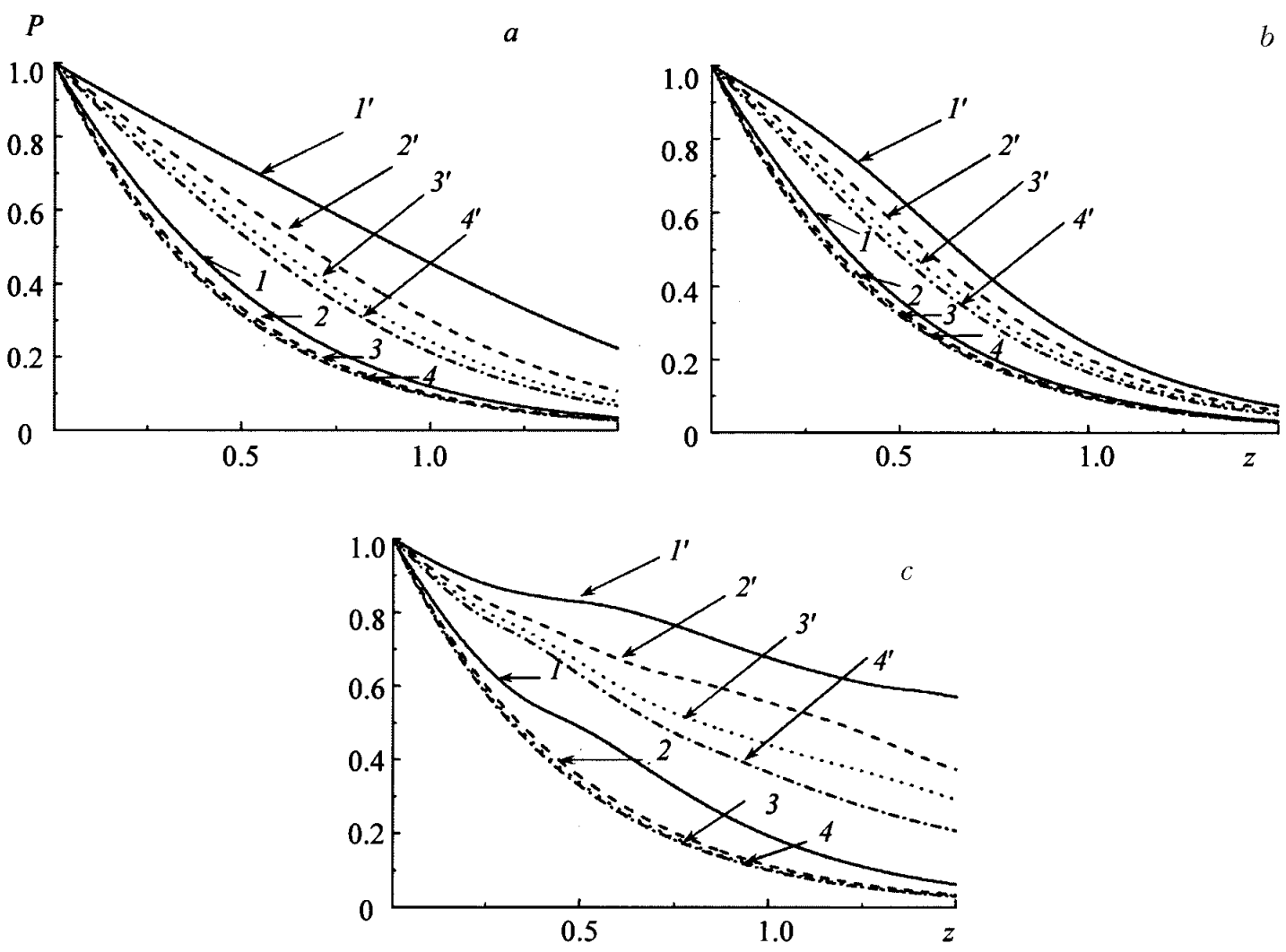

Fig. 2. Light-beam power as a function of penetration depth into a nonlinear medium for $k=0.05 \mathrm{~cm}^{-1} ; r_{0}=200 \mu \mathrm{m} ; \Delta=0$ (a), -2 (b), 2 (c); $\alpha I_{0}=1$ $(1,2), 0.5$ (3), $0.167(4), 10\left(1^{\prime}, 2^{\prime}\right), 5\left(3^{\prime}\right), 1.67\left(4^{\prime}\right) ; m=0\left(1,1^{\prime}\right), 1\left(2,2^{\prime}\right)$, $2\left(3,3^{\prime}\right), 3\left(4,4^{\prime}\right)$.

Figure $2 \mathrm{c}$ shows the case of focusing nonlinearity occurring with a positive frequency detuning from the center of the resonant transition. Conditions sufficient for inducing self-focusing of the radiation are fulfilled for the selected parameters of the Gaussian beam at a peak intensity of the order of the saturation intensity of the resonant transition $\alpha I_{0} \sim 1$. Thus, an inflection point near $z \sim 0.5$ is observed on the function $P(z)$ (curve 1) that reflects a local decrease of the absorption coefficient of the nonlinear medium near the greatest focusing of the radiation. As the input intensity of the light beam increases further, multi-focused self-focusing that is evident as the presence of several inflections on the function $P(z)$ (curve 1') may be observed. The self-focusing is evident for singular light beams at a high input intensity (curves $2^{\prime}$ and $4^{\prime}$ ). However, as shown below, the transverse structure of such beams undergoes more complex changes.

Formation of vortex solitons under defocusing nonlinearity conditions. A nonlinear medium in which the index of refraction decreases with increasing intensity of the light beam can promote the formation of so-called dark transverse solitons [21] that are characterized by localized gaps in the intensity of the transverse profile. Self-channeling of the vortex structure of the wave front of a singular beam under such conditions leads to the formation of vortex solitons, which have been observed experimentally in a medium with thermal nonlinearity [22], in rubidium vapor [23], and in photorefractive crystals [24].

The numerical calculations show that the formation and destruction of the dark localized structure occurs in several stages (Fig. 3 and Fig. 4a) for propagation of a singular light beam with a single topological charge in a resonant medium with defocusing nonlinearity. A sharp narrowing of the singularity region $\left(\Delta_{\mathrm{T}(0.5)}\right.$ is the half-width of the dark region at half intensity) that depends on the intensity occurs first and at $z \sim 0.2$, formation of a dark soliton and approximate retention of its shape in the range $z \sim 0.2-0.3$. We note that the calculated profile of the light beam at 

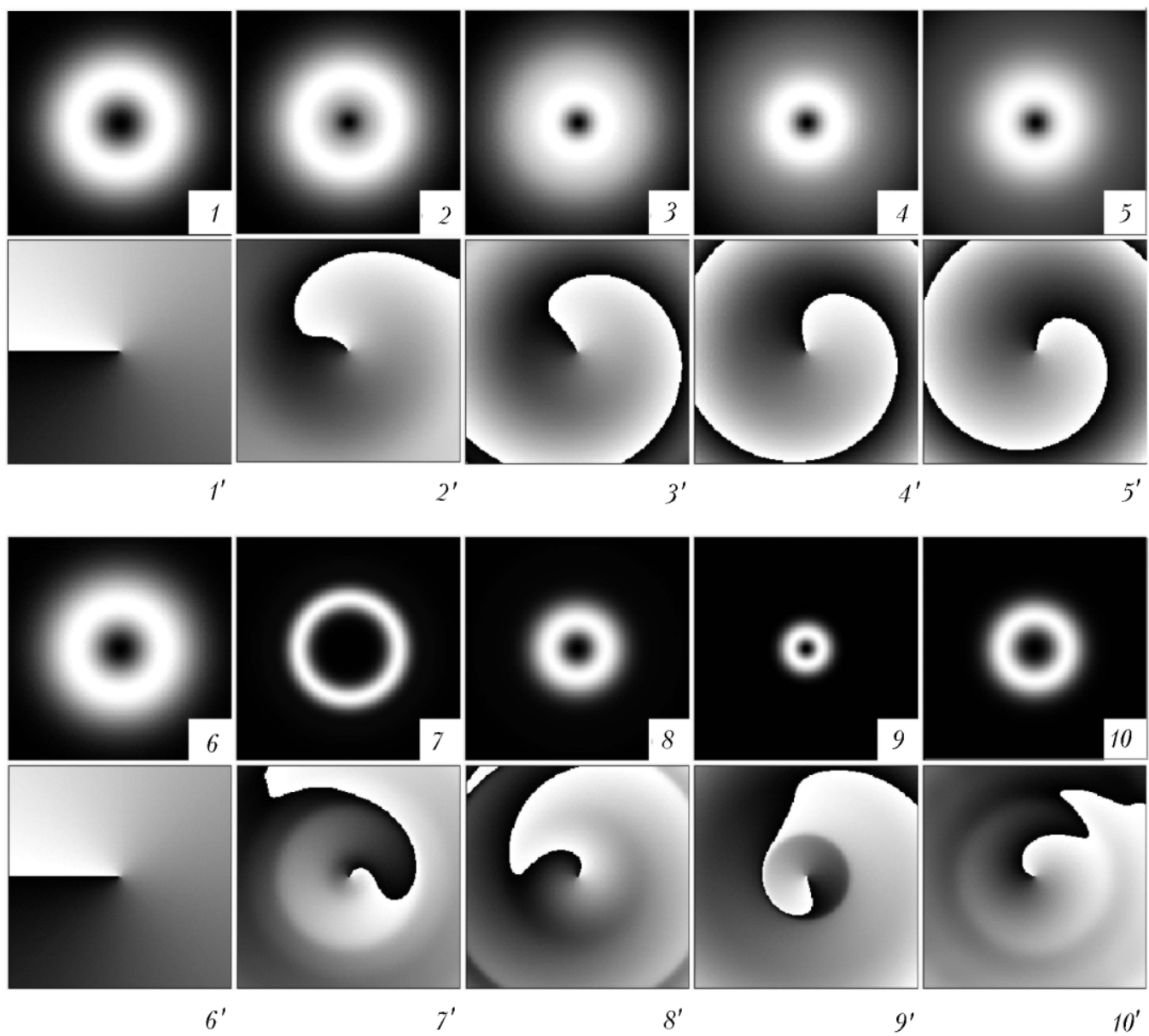

Fig. 3. Intensity distribution and wave front of optical vortices for $k=0.05$ $\mathrm{cm}^{-1} ; r_{0}=200 \mu \mathrm{m} ; m=1 ; z=0$ (1), 0.1 (2), 0.2 (3), 0.3 (4), 0.4 (5); 0 (6), 0.25 (7), 0.5 (8), 0.75 (9), $1(10) ; \Delta=-2\left(1-5,1^{\prime}-5^{\prime}\right), 2\left(6-10,6^{\prime}-10^{\prime}\right) ; \alpha I_{0}$ $=10\left(1-5,1^{\prime}-5^{\prime}\right), 5\left(6-10,6^{\prime}-10^{\prime}\right)$.

$z=0.3$ is shown by a solid line in the inset in Fig. $4 \mathrm{a}$ whereas the dashed line shows the intensity distribution over the profile of the dark soliton $I_{\mathrm{TC}}=I_{1} \tan ^{2}\left(x / a_{1}\right)$ [25], which can be obtained by solving the nonlinear Schrödinger equation for Kerr-type defocusing nonlinearity. Upon further propagation, the light beam loses power as a result of absorption and the region of localization experiences diffraction spreading. The wave-front surface of the singular beam is distorted also at all stages of propagation (Fig. 3, $1^{\prime}-5^{\prime}$ ) because of the nonlinear modulation of the index of refraction in the area where the energy of the light beam is localized.

Self-focusing of optical vortices. The change of transverse structure of singular light beams in the self-focusing regime (Fig. 3 and Fig. 4b) is determined by competition of nonlinear compression and diffraction spreading. Thus, multi-focusing regimes of transverse structure modulation can be observed. We will introduce the parameters $\Delta_{\mathrm{V}(0.5)}$ and $\Delta_{\mathrm{R}(0.5)}$ that determine the half-width of the optical vortex as a whole and the half-width of its light ring at half intensity. Focusing of the light ring occurs in the first stage $(z=0-0.25)$ and, therefore, $\Delta_{\mathrm{R}(0.5)}$ decreases (Fig. 4b, curve 2). Thus, the half-width of the optical vortex $\Delta_{\mathrm{V}(0.5)}$ (curve $2^{\prime}$ ) increases slightly. Then, the reverse process occurs that is replaced by a general self-focusing of the singular beam after $z=0.5$. The increase of the peak intensity of the light beam near the nonlinear focus at $z=0.75$ leads to saturation of the resonant transition and a substantial decrease of the absorption near the beam localization. This enables the localized structure to be self-sustained upon propagation in the nonlinear medium from $z=0.6$ to $z=0.8$. Then, diffraction spreading of the light beam becomes the dominant process. 

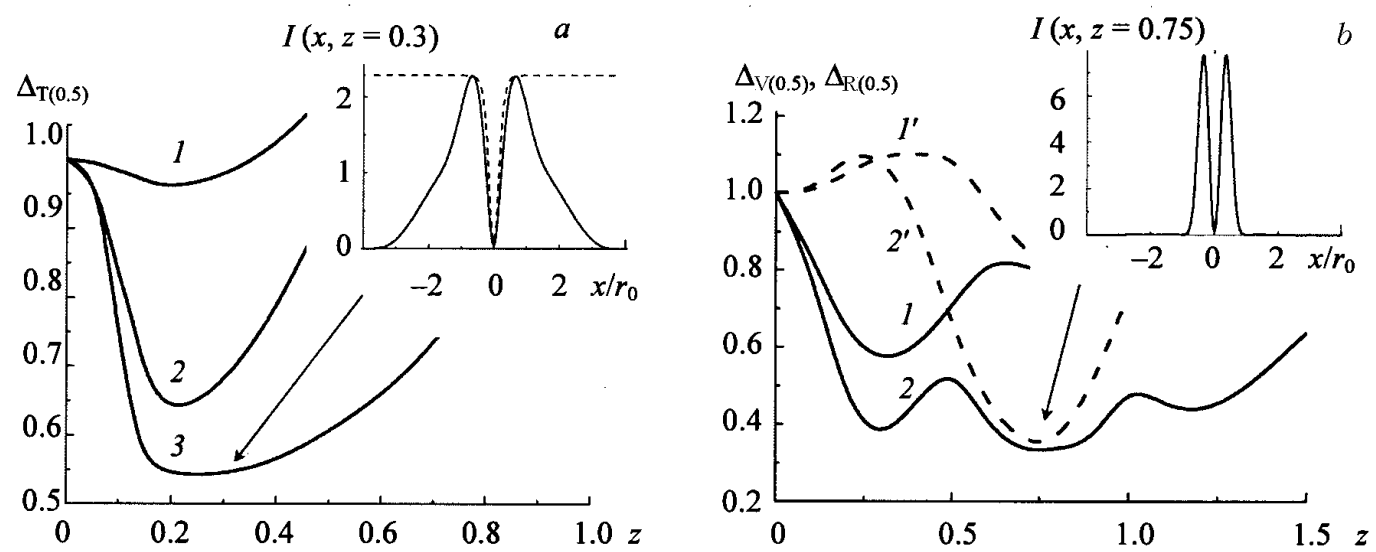

Fig. 4. Light beam half-width as a function of penetration depth into a nonlinear medium for $k=0.05 \mathrm{~cm}^{-1} ; r_{0}=200 \mu \mathrm{m}$; and $m=1 . \Delta=-2 ; \alpha I_{0}=$ 0.1 (1), 1 (2), 10 (3) (a); $\Delta=2, \alpha I_{0}=1\left(1,1^{\prime}\right), 10$ (2, 2') (b); insets show profiles of light beams in the nonlinear medium bulk.

The numerical calculations show that this scenario of transverse evolution of singular light beams under selffocusing conditions in resonant media is not the only one possible. Its transverse structure can be destroyed if the initial beam is additionally modulated under certain conditions. This is analogous to the situation examined previously [26] of purely dispersive nonlinearity. However, a detailed investigation of this issue is beyond the scope of this article.

Conclusion. Peculiarities of the transformation of the transverse structure of light beams with a vortex dislocation of the wave front are analyzed based on numerical solution of a truncated wave equation. The conditions for self-focusing and defocusing of singular light beams in resonant media are determined. It is shown that self-modulation of light beams as the result of nonlinear effects in the change of absorption coefficient and index of refraction of the resonant medium can lead to the formation of self-localized transverse structures as dark solitons.

\section{REFERENCES}

1. J. F. Nye and M. V. Berry, Proc. R. Soc. London, A, 336, 165-190 (1974).

2. B. Ya. Zel'dovich, N. F. Pilipetskii, and V. V. Shkunov, Wave Front Inversion [in Russian], Nauka, Moscow (1985).

3. M. Brambilla, F. Battipede, L. A. Lugiato, V. Penna, F. Pratti, C. Tamm, and C. O. Weiss, Phys. Rev. A, 43, 5090-5117 (1991).

4. N. R. Heckenberg, R. McDuff, S. P. Smith, and A. G. White, Opt. Lett., 17, 221-223 (1992).

5. A. Ya. Bekshaev, M. S. Soskin, and M. V. Vasnetsov, Opt. Commun., 241, 237-247 (2004).

6. A. Desyatnikov, D. Mihalache, D. Mazilu, B. Malomed, and F Lederer, Phys. Lett. A, 364, 231-234 (2007).

7. V. I. Kruglov and R. A. Vlasov, Phys. Lett. A, 111, 401-404 (1985).

8. Yu. S. Kivshar and B. Luther-Davies, Phys. Rep., 298, 81-197 (1998).

9. D. V. Skryabin and W. J. Firth, Phys. Rev. E: Stat. Phys., Plasmas, Fluids, Relat. Interdiscip. Top., 58, 39163930 (1998).

10. O. Angelsky, A. Mokhun, I. Mokhun, and M. Soskin, Opt. Commun., 207, 57-65 (2002).

11. C Lopez-Mariscal, J. Gutierrez-Vega, D. McGloin, and K. Dholakia, Opt. Express, 15, 6330-6335 (2007).

12. W. Jiang, Q. Chen, Y. Zhang, and G.-C. Guo, Phys. Rev. A, 74, 043811-1-043811-4 (2006).

13. N. S. Kazak, E. G. Katranzhi, I. A. Utkin, A. A. Ryzhevich, and A. N. Khilo, Zh. Prikl. Spektrosk., 71, No. 5, 640-643 (2004).

14. P. I. Ropot and R. Yu. Vasil'ev, Zh. Prikl. Spektrosk., 73, No. 4, 488-493 (2006).

15. B. B. Sevruk, Zh. Prikl. Spektrosk., 73, No. 5, 626-630 (2006).

16. I. R. Shen, Principles of Nonlinear Optics [in Russian], Nauka, Moscow (1989). 
17. V. V. Kabanov and A. S. Rubanov, Dokl. Akad. Navuk BSSR, 24, 34-37 (1980).

18. R. H. Pantell and H. E. Puthoff, Fundamentals of Quantum Electronics, Wiley, New York (1969).

19. V. K. Saul'ev, Integration of Equations of Parbolic Type by the Method of Nets [in Russian], Fizmatgiz, Moscow (1960).

20. V. L. Derbov, L. A .Melnikov, A. D. Novikov, and S. K. Potapov, J. Opt. Soc. Am. B: Opt. Phys., 7, 10791097 (1990).

21. Yu. S. Kivshar' and G. P. Agraval, Optical Solitons. From Fiber Lightguides to Photonic Crystals [in Russian], Fizmatlit, Moscow (2005).

22. G. A. Swartzlander, Jr., and C. Law, Phys. Rev. Lett., 69, 2503-2506 (1992).

23. V. Tikhonenko, J. Christou, B. Luther-Davies, and Yu. S. Kivshar, Opt. Lett., 21, 1129-1131 (1996).

24. Z. Chen, M. Shih, M. Segev, D. W. Wilson, R. E. Muller, and P. D. Maker, Opt. Lett., 22, 1751-1753 (1997).

25. W. J. Tomlinson, R. J. Hawkins, A. M. Weiner, J. P. Heritage, and R. N. Thurston, J. Opt. Soc. Am. B: Opt. Phys., 6, 329-334 (1989).

26. A. Dreischuh, G. G. Paulus, F. Zacher, F. Grasbon, D. Neshev, and H. Walther, Phys. Rev. E: Stat. Phys., Plasmas, Fluids, Relat. Interdiscip. Top., 60, 7518-7524 (1999). 